Real Analysis Exchange

Vol. 22(1), 1996-97, pp. 382-389

Lee Tuo-Yeong and Chew Tuan-Seng, Department of Mathematics, National University of Singapore, Singapore 119260

Lee Peng-Yee, Mathematics Division, National Institute of Education, Bukit Timah Road, Singapore 259756

\title{
ON HENSTOCK INTEGRABILITY IN EUCLIDEAN SPACES
}

\begin{abstract}
In this paper, we give a necessary and sufficient condition in terms of Lebesgue integrable functions for Henstock integrability in Euclidean space.
\end{abstract}

By means of the Cauchy and Harnack extension theorems for the onedimensional Henstock integral, Liu [5] proved that

Theorem 1 If $f$ is Henstock integrable on $[a, b]$, then there is a sequence $\left\{X_{k}\right\}$ of closed subsets of $[a, b]$ such that $X_{k} \subset X_{k+1}$ for all $k, \bigcup_{k=1}^{\infty} X_{k}=[a, b], f$ is Lebesgue integrable on each $X_{k}$ and

$$
\lim _{k \rightarrow \infty}(L) \int_{X_{k} \cap[a, x]} f(t) d t=(H) \int_{a}^{x} f(t) d t
$$

uniformly on $[a, b]$.

Liu's proof is real-line dependent, and so it is difficult to generalize Theorem 1 to higher dimensions. In this note, we shall give a direct proof of the multidimensional version of Liu's result. Consequently, we deduce a necessary and sufficient condition for Henstock integrability in higher dimensions (Theorem 7).

First, we give some preliminaries (see [3]).

Let $\mathbf{R}$ and $\mathbf{R}^{+}$denote the real line and the positive real line respectively, $m$ a fixed positive integer and $\mathbf{R}^{m}$ the $m$-dimensional euclidean space.

Mathematical Reviews subject classification: Primary: 26A39

Received by the editors January 1, 1996 
Unless otherwise stated, an interval will always be a compact nondegenerate interval of the form $[s, t]=\prod_{i=1}^{m}\left[s_{i}, t_{i}\right]$ where $s=\left(s_{1}, s_{2}, \ldots, s_{m}\right)$ and $t=\left(t_{1}, t_{2}, \ldots, t_{m}\right)$.

Also, $E=\prod_{i=1}^{m}\left[a_{i}, b_{i}\right]$ will denote a fixed interval in $\mathbf{R}^{m}$, and $B(x, \delta)$ denotes an open ball in $\mathbf{R}^{m}$ with center $x$ and radius $\delta$. A finite collection of intervals whose interiors are disjoint is called a nonoverlapping collection. A partial division $D=\{(I, \xi)\}$ of $E$ is a finite collection of interval-point pairs such that the collection of intervals are non-overlapping. If, in addition, the union of $I$ from $D$ gives $E$, we say that $D$ is a division of $E$. Let $\delta: E \longrightarrow \mathbf{R}^{+}$ be given. A partial division $D=\{(I, \xi)\}$ is said to be $\delta$-fine if for each $(I, \xi) \in D$ with $\xi$ being a vertex of $I$, we have $I \subset B(\xi, \delta(\xi))$.

In this note, all functions will be assumed to be real-valued, and often the same letter is used to denote a function on $E$ as well as its restriction to a set $Z \subset E$. A function $f: E \longrightarrow \mathbf{R}$ is said to be Henstock integrable to a real number $A$ on $E$ if for every $\varepsilon>0$, there exists $\delta: E \longrightarrow \mathbf{R}^{+}$such that for any $\delta$-fine division $D=\{(I, \xi)\}$ of $E$, we have

$$
\left|(D) \sum f(\xi)\right| I|-A|<\varepsilon \text {. }
$$

We write $A=(H) \int_{E} f$. If $g$ is Lebesgue integrable on $E$, we write the Lebesgue integral of $g$ over $E$ as $(L) \int_{E} g$. It is known that if $g$ is Lebesgue integrable on $E$, then $g$ is Henstock integrable there with the same integral value. For a proof, see [6, Proposition 4, Remark 6]. The words "measure", "measurable" and "almost everywhere" always refer to the $m$-dimensional Lebesgue measure. If $X$ is measurable, we shall write $|X|$ as the $m$-dimensional Lebesgue measure of $X$. We next give Henstock's lemma.

Theorem 2 If $f$ is Henstock integrable on $E$, then for every $\varepsilon>0$, there exists $\delta: E \longrightarrow \mathbf{R}^{+}$such that for any $\delta$-fine partial division $D=\{(I, \xi)\}$ of $E$, we have

$$
\text { (D) } \sum|f(\xi)| I\left|-(H) \int_{I} f\right|<\varepsilon .
$$

As a consequence of Henstock's lemma, we shall prove the following two lemmas.

Lemma 3 If $f$ is Henstock integrable on $E$, then for every $\epsilon>0$, there exists $\delta: E \longrightarrow \mathbf{R}^{+}$such that for every $\delta$-fine partial division $D=\{(I, \xi)\}$ of $E$, we have

$$
\text { (D) } \sum|f(\xi)| I \cap E_{0}\left|-(H) \int_{I \cap E_{0}} f\right|<\varepsilon
$$

for every subinterval $E_{0}$ of $E$. 
Proof. By Theorem 2, for $\varepsilon>0$, there exists $\delta: E \longrightarrow \mathbf{R}^{+}$such that for any $\delta$-fine partial division $D=\{(I, \xi)\}$ of $E$, we have

$$
\text { (D) } \sum|f(\xi)| I\left|-(H) \int_{I} f\right|<\frac{\varepsilon}{2^{m+1}} .
$$

Let $E_{0}$ be a subinterval of $E$. We let

$$
D_{0}=\left\{\left(I \cap E_{0}, \xi\right):\left|I \cap E_{0}\right|>0 \text { and }(I, \xi) \in D\right\} .
$$

Writing $S=(D) \sum|f(\xi)| I \cap E_{0}\left|-(H) \int_{I \cap E_{0}} f\right|$, we want to show that $S<\varepsilon$. Note that

$$
\begin{aligned}
S= & (D) \sum|f(\xi)| I \cap E_{0}\left|-(H) \int_{I \cap E_{0}} f\right| \\
= & \left(D_{0}\right) \sum|f(\xi)| I \cap E_{0}\left|-(H) \int_{I \cap E_{0}} f\right| \\
= & \left(D_{0}\right) \sum_{\xi \in E_{0}}|f(\xi)| I \cap E_{0}\left|-(H) \int_{I \cap E_{0}} f\right| \\
& \quad+\left(D_{0}\right) \sum_{\xi \notin E_{0}}|f(\xi)| I \cap E_{0}\left|-(H) \int_{I \cap E_{0}} f\right| \\
& <\frac{\varepsilon}{2^{m+1}}+\left(D_{0}\right) \sum_{\xi \notin E_{0}}|f(\xi)| I \cap E_{0}\left|-(H) \int_{I \cap E_{0}} f\right|
\end{aligned}
$$

as $\left\{\left(I \cap E_{0}, \xi\right): \xi \in E_{0}\right.$ and $\left.(I, \xi) \in D\right\}$ is a $\delta$-fine partial division of $E$. Hence we have

$$
S<\frac{\varepsilon}{2^{m+1}}+\left(D_{0}\right) \sum_{\xi \notin E_{0}}|f(\xi)| I \cap E_{0}\left|-(H) \int_{I \cap E_{0}} f\right|
$$

It remain to prove that the second term in (2) is less than $\frac{\varepsilon}{2}$. Note that when $\xi \notin E_{0}$, the interval $I \cap E_{0}$ does not contain $\xi$ and therefore $\left(I \cap E_{0}, \xi\right)$ is no longer $\delta$-fine.

Let $D_{1}=\left\{\left(I \cap E_{0}, \xi\right) \in D_{0}: \xi \notin E_{0}\right.$ and $\left.(I, \xi) \in D\right\}=\left\{\left(I_{j} \cap E_{0}, \xi_{j}\right)\right\}_{j=1}^{p}$ and for each subinterval $E_{1}$ of $E$, we put

$$
G_{j}\left(I_{j} \cap E_{1}, \xi_{j}\right)=f\left(\xi_{j}\right)\left|I_{j} \cap E_{1}\right|-(H) \int_{I_{j} \cap E_{1}} f
$$

for each $j=1,2, \ldots, p$. 
We recall that if $x=\left(x_{1}, x_{2}, \ldots, x_{m}\right)$ and $y=\left(y_{1}, y_{2}, \ldots, y_{m}\right)$ are two distinct vertices of an interval $I, x$ and $y$ are said to be opposite if $x_{i} \neq y_{i}$ for all $i=1,2, \ldots, m$. We shall denote an interval with $\xi, x$ as opposite vertices by $\langle\xi, x>$. Then for each $j=1,2, \ldots, p$,

$$
\begin{aligned}
& \left|f\left(\xi_{j}\right)\right| I_{j} \cap E_{0}\left|-(H) \int_{I_{j} \cap E_{0}} f\right|=\left|G_{j}\left(I_{j} \cap E_{0}, \xi_{j}\right)\right| \text { by }(3) \\
& =\left|\sum_{l=1}^{2^{m}}(-1)^{n(l, j)} G_{j}\left(<\xi_{j}, \gamma^{(l, j)}>, \xi_{j}\right)\right| \leq \sum_{l=1}^{2^{m}}\left|G_{j}\left(<\xi_{j}, \gamma^{(l, j)}>, \xi_{j}\right)\right|
\end{aligned}
$$

where $\gamma^{(l, j)}=\left(\gamma_{1}^{(l, j)}, \gamma_{2}^{(l, j)}, \ldots, \gamma_{m}^{(l, j)}\right)$ represents a vertex of $I_{j} \cap E_{0}$ $=\prod_{i=1}^{m}\left[\alpha_{i}^{(j)}, \beta_{i}^{(j)}\right]$ and $n(l, j)$ is the cardinality of the set $\left\{i: \gamma_{i}^{(l, j)}=\alpha_{i}^{(j)}\right\}$.

Hence, for $j=1,2, \ldots, p$,

$$
\left|G_{j}\left(I_{j} \cap E_{0}, \xi_{j}\right)\right| \leq \sum_{l=1}^{2^{m}}\left|G_{j}\left(<\xi_{j}, \gamma^{(l, j)}>, \xi_{j}\right)\right|
$$

Consequently, by (4),

$$
\begin{aligned}
\sum_{j=1}^{p} & \left|f\left(\xi_{j}\right)\right| I_{j} \cap E_{0}\left|-(H) \int_{I_{j} \cap E_{0}} f\right|=\sum_{j=1}^{p}\left|G_{j}\left(I_{j} \cap E_{0}, \xi_{j}\right)\right| \\
\leq & \sum_{j=1}^{p} \sum_{l=1}^{2^{m}}\left|G_{j}\left(<\xi_{j}, \gamma^{(l, j)}>, \xi_{j}\right)\right|=\sum_{l=1}^{2^{m}} \sum_{j=1}^{p}\left|G_{j}\left(<\xi_{j}, \gamma^{(l, j)}>, \xi_{j}\right)\right|
\end{aligned}
$$

Recall that $D_{0}=\left\{\left(I \cap E_{0}, \xi\right):\left|I \cap E_{0}\right|>0\right.$ and $\left.(I, \xi) \in D\right\}$ and $D_{1}=$ $\left\{\left(I \cap E_{0}, \xi\right) \in D_{0}: \xi \notin E_{0}\right.$ and $\left.(I, \xi) \in D\right\}=\left\{\left(I_{j} \cap E_{0}, \xi_{j}\right)\right\}_{j=1}^{p}$. By our definition of $D_{0}$ and $D_{1}$, we see that $\left\{\left(<\xi_{j}, \gamma^{(l, j)}>, \xi_{j}\right)\right\}_{j=1}^{p}$ is a $\delta$-fine partial division of $E$ for each $l=1,2, \ldots, 2^{m}$. We have

$$
\begin{aligned}
& \sum_{j=1}^{p}\left|f\left(\xi_{j}\right)\right| I_{j} \cap E_{0}\left|-(H) \int_{I_{j} \cap E_{0}} f\right| \leq \sum_{l=1}^{2^{m}} \sum_{j=1}^{p}\left|G_{j}\left(<\xi_{j}, \gamma^{(l, j)}>, \xi_{j}\right)\right| \\
& \quad<\sum_{l=1}^{2^{m}} \frac{\varepsilon}{2^{m+1}} \text { by Theorem } 2 \\
& \quad=\frac{\varepsilon}{2}
\end{aligned}
$$

By (2), we get the required inequality. The proof is complete. 
Lemma 4 Suppose $f$ is Henstock integrable on $E$, and $f$ is Lebesgue integrable on some closed subset $Y$ of $E$. Then given $\epsilon>0$, there exists $\delta: Y \longrightarrow \mathbf{R}^{+}$ such that for any $\delta$-fine partial division $D=\{(I, \xi)\}$ with $\xi \in Y$, we have

$$
(D) \sum\left|(L) \int_{I \cap Y \cap E_{0}} f-(H) \int_{I \cap E_{0}} f\right|<\varepsilon
$$

for every subinterval $E_{0}$ of $E$.

Proof. By Lemma 3, there exists $\delta_{1}: E \longrightarrow \mathbf{R}^{+}$such that for any $\delta_{1}$-fine partial division $D=\{(I, \xi)\}$ of $E$, we have

$$
(D) \sum|f(\xi)| I \cap E_{0}\left|-(H) \int_{I \cap E_{0}} f\right|<\frac{\varepsilon}{2}
$$

for every subinterval $E_{0}$ of $E$.

Since $f$ is Lebesgue integrable on $Y, f \chi_{Y}$ is Henstock integrable on $E$, where $\chi_{Y}$ denotes the characteristic function of $Y$. So there exists $\delta_{2}: E \longrightarrow$ $\mathbf{R}^{+}$such that for any $\delta_{2}$-fine partial division $D=\{(I, \xi)\}$ of $E$ with $\xi \in Y$, we have

$$
(D) \sum\left|f(\xi) \chi_{Y}(\xi)\right| I \cap E_{0}\left|-(L) \int_{I \cap E_{0}} f \chi_{Y}\right|<\frac{\varepsilon}{2}
$$

for every subinterval $E_{0}$ of $E$.

Define $\delta: Y \longrightarrow \mathbf{R}^{+}$as follows: $\delta(\xi)=\min \left\{\delta_{1}(\xi), \delta_{2}(\xi)\right\}$. Then for any $\delta$-fine partial division $D=\{(I, \xi)\}$ of $E$ with $\xi \in Y$, it is both $\delta_{1}$-fine and $\delta_{2}$-fine. Thus

$$
\begin{aligned}
& (D) \sum\left|(L) \int_{I \cap Y \cap E_{0}} f-(H) \int_{I \cap E_{0}} f\right| \\
& \leq(D) \sum\left|(L) \int_{I \cap E_{0}} f \chi_{Y}-f(\xi)\right| I \cap E_{0}|| \\
& \quad+(D) \sum|f(\xi)| I \cap E_{0}\left|-(H) \int_{I \cap E_{0}} f\right| \\
& \quad<\varepsilon \text {, by (5) and (6). }
\end{aligned}
$$

The proof is complete.

The next theorem due to Kurzweil and Jarnik [2, Theorem 2.10] is also an important tool. For convenience, in what follows, we shall write $X_{k} \uparrow E$ to mean $X_{k} \subset X_{k+1}$ for all $k$ and $\bigcup_{k=1}^{\infty} X_{k}=E$. 
Theorem 5 Let $f$ be Henstock integrable on $E$. Then there exists a sequence $\left\{Y_{k}\right\}$ of closed sets with $Y_{k} \uparrow E$ and $f$ is Lebesgue integrable on each $Y_{k}$.

We shall now state and prove the multidimensional version of Liu's result.

Theorem 6 If $f$ is Henstock integrable on the interval $E$, then there exists a sequence $\left\{X_{k}\right\}$ of closed subsets of $E$ such that $X_{k} \uparrow E, f$ is Lebesgue integrable on each $X_{k}$ and

$$
\sup \left|(L) \int_{X_{k} \cap E_{1}} f-(H) \int_{E_{1}} f\right| \leq \frac{1}{k}
$$

for each $k$, and the above supremum is over all subintervals $E_{1}$ of $E$.

Proof. In view of Theorem 5, there exists a sequence $\left\{Y_{k}\right\}$ of closed sets with $Y_{k} \uparrow E$ and $f$ is Lebesgue integrable on each $Y_{k}$. By Lemma 4, for every positive integer $\mathrm{n}$ and for each $\mathrm{k}$ there exists $\delta_{k}: Y_{k} \longrightarrow \mathbf{R}^{+}$such that for any $\delta_{k}$-fine partial division $D=\{(I, \xi)\}$ of $E$ with $\xi \in Y_{k}$, we have

$$
(D) \sum\left|(L) \int_{I \cap Y_{k} \cap E_{0}} f-(H) \int_{I \cap E_{0}} f\right|<\frac{1}{2^{k} n}
$$

for every subinterval $E_{0}$ of $E$. Next we want to choose $\left\{X_{n}\right\}$ from $\left\{Y_{k}\right\}$ so that the required inequality holds. By our assumption, $Y_{k}$ is closed, so $\operatorname{dist}\left(\xi, Y_{k}\right)$ $>0$ if and only if $\xi \notin Y_{k}$ where $\operatorname{dist}\left(\xi, Y_{k}\right)$ denotes the distance between $\xi$ and $Y_{k}$. Define $\delta: E \longrightarrow \mathbf{R}^{+}$as follows :

$$
\delta(\xi)= \begin{cases}\delta_{n}(\xi), & \text { if } \xi \in Y_{n} \\ \min \left\{\delta_{k}(\xi), \operatorname{dist}\left(\xi, Y_{k-1}\right)\right\}, & \text { if } \xi \in Y_{k}-Y_{k-1} \quad \text { for each } k>n .\end{cases}
$$

Since a $\delta$-fine division of $E$ exists, see for example [3, p. 128], we may fix a $\delta$-fine division $D_{0}=\{(I, \xi)\}$ of $E$. For simplicity, we put $P_{n}=Y_{n}$ and $P_{k}=Y_{k}-Y_{k-1}$ for $k>n$. Next, we put

$$
X_{n}=\bigcup_{j=n}^{\infty}\left\{I \cap Y_{j}:(I, \xi) \in D_{0} \text { with } \xi \in P_{j}\right\}
$$

The above union is a finite one because $D_{0}$ only has finitely many terms. Thus $X_{n}$ is closed as each $Y_{j}$ is closed.

Define

$$
k(n)=\max \left\{j:(I, \xi) \in D_{0} \text { and } \xi \in P_{j}\right\}+1
$$

Since $Y_{k} \uparrow E$, we have

$$
Y_{k(n)} \supseteq X_{n}
$$


By the definition of $\delta$ and the compactness of $Y_{n}$, any $\delta$-fine division $D=$ $\{(I, \xi)\}$ must cover $Y_{n}$. Hence

$$
Y_{n} \subseteq X_{n}
$$

By (10) and (11), we have

$$
Y_{n} \subseteq X_{n} \subseteq Y_{k(n)} .
$$

By (12), we note that $f$ is Lebesgue integrable on $X_{n}$. of $E$.

Claim. $\left|(L) \int_{E_{1} \cap X_{n}} f-(H) \int_{E_{1}} f\right| \leq \frac{1}{n}$ for every closed subinterval $E_{1}$ by (8),

Observe that if $(I, \xi) \in D_{0}$ with $\xi \in P_{l}$ for some positive integer $l$, then

$$
I \cap X_{n}=I \cap Y_{l} .
$$

Note that $D_{0}$ may have its associated points belonging to $P_{n}$ only. Without loss of generality, we may suppose $D_{0}$ has its associated points belonging to

$$
P_{s_{1}}, P_{s_{2}}, \ldots, P_{s_{l}}
$$

for some positive integers $s_{1}<s_{2}<\cdots<s_{l}$ with $s_{1}=n$. Now, we have for all closed subintervals $E_{1}$ of $E$,

$$
\begin{aligned}
\mid(L) \int_{E_{1} \cap X_{n}} f & -(H) \int_{E_{1}} f\left|\leq\left(D_{0}\right) \sum\right|(L) \int_{E_{1} \cap I \cap X_{n}} f-(H) \int_{E_{1} \cap I} f \mid \\
& \leq \sum_{i=1}^{l}\left(D_{0}\right) \sum_{\xi \in P_{s_{i}}}\left|(L) \int_{E_{1} \cap I \cap X_{n}} f-(H) \int_{E_{1} \cap I} f\right| \\
& =\sum_{i=1}^{l}\left(D_{0}\right) \sum_{\xi \in P_{s_{i}}}\left|(L) \int_{E_{1} \cap I \cap Y_{s_{i}}} f-(H) \int_{E_{1} \cap I} f\right| \text { by (13) } \\
& <\frac{1}{n} \text { by }(7) .
\end{aligned}
$$

It is easy to see from (12) that there exists a subsequence of $\left\{X_{n}\right\}$, denoted again by $\left\{X_{n}\right\}$, such that $X_{n} \uparrow E$. The proof is complete.

We remark that Theorem 6 is indeed a generalization of Liu's result. Furthermore, Theorem 7 below is also an improvement of the results in Liu [5] and Lee [4]. 
Theorem 7 A function $f$ is Henstock integrable on $E$ if and only if there exists a sequence $\left\{X_{k}\right\}$ of closed subsets of $E$ such that $X_{k} \uparrow E, f$ is Lebesgue integrable on each $X_{k}$ and the following condition holds: for every $\epsilon>0$ there exists an integer $N$ such that if $k \geq N$ then there exists $\delta_{k}: E \longrightarrow \mathbf{R}^{+}$such that for every $\delta_{k}$-fine division $D=\{(I, \xi)\}$ of $E$ we have

$$
\left|(D) \sum_{\xi \notin X_{k}} f(\xi)\right| I \cap E_{1}||<\epsilon
$$

for every subinterval $E_{1}$ of $E$.

Proof. The proof follows easily as in Bartle [1], by taking note that

$$
\text { (D) } \sum_{\xi \notin X_{k}}=(D) \sum-(D) \sum_{\xi \in X_{k}}
$$

and using Theorem 6 .

\section{References}

[1] R. G. Bartle, A convergence theorem for generalized Riemann integrals, Real Analysis Exchange, 20 (1994-95), 119-124.

[2] J. Kurzweil and J. Jarnik, Equiintegrability and Controlled Convergence of Perron-type integrable functions, Real Analysis Exchange, 17 (1991-92), 110-139.

[3] L. Peng-Yee, Lanzhou lectures on Henstock integration, World Scientific, 1989.

[4] L. Peng-Yee, Measurability and the Henstock integral, Proc. Internat. Math. Conf., 1994, Kaohsiung, World Scientific, 1995.

[5] Liu Genqian, On necessary conditions for Henstock integrability, Real Analysis Exchange, 18 (1992-93), 522-531.

[6] W. F. Pfeffer, A note on the generalized Riemann integral, Proc. American Math Soc., 103 (1988), 1161-1166. 